\title{
A predator-prey optimization for structural health monitoring problems
}

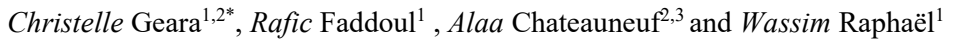 \\ ${ }^{1}$ ESIB, Université Saint-Joseph, Mar Roukos, PoB. 11-154, Riad El Solh, Beyrouth, Liban \\ ${ }^{2}$ Université Clermont Auvergne, CNRS, Institut Pascal, 63000 Clermont-Ferrand, France \\ ${ }^{3}$ CIDECO, 2 av. Blaise Pascal, 63178 Aubière, France
}

\begin{abstract}
Monitoring a structure using permanent sensors has been one of the most interesting topics, especially with the increase of the number of aging structures. Such a technique requires the implementation of sensors on a structure to predict the condition states of the structural elements. However, due to the costs of sensors, one must judiciously install few sensors at some defined locations in order to maximize the probability of detecting potential damages. In this paper, we propose a methodology based on a genetic algorithm of type predator-prey with a Bayesian updating of the structural parameters, to optimize the number and location of the sensors to be placed. This methodology takes into consideration all uncertainties related to the degradation of the elements, the mechanical model and the accuracy of sensors. Starting with two initial populations representing the damages (prey) and the sensors (predator), the genetic algorithm evolves both populations in order to converge towards the optimal configuration of sensors, in terms of number and location. The proposed methodology is illustrated by a two-story concrete frame structure.
\end{abstract}

\section{Introduction}

Following the growing expansion of civil engineering infrastructures and the aging of some structures, the Structural Health Monitoring (SHM) is currently given a great and particular importance [1-3]. The SHM combines a variety of sensing technologies for the detection and localization of damages in order to assess the state of the structure and predict its residual life. However, limited budgets are given for the monitoring, maintenance and rehabilitation of structures and infrastructures. For example, according to the European Investment Bank (EIB), public investment in infrastructure is at its lowest level in 20 years (EIB Investment Report 2017/2018). Therefore, a cost-effective plan is needed in order to monitor structures and follow their state of degradation through the years.

Structural health monitoring can be divided into two approaches: (i) The Local SHM based on a direct evaluation of an element or a part of a structure to evaluate its state [4] and (ii) the Global SHM based on a mechanical modeling of the structure where few sensors (whose number and locations are to be optimized) are used to monitor the whole structure [5]. The advantage of using the indirect methods of the global SHM lies in their ability to take into consideration, systematically, all the uncertainties affecting the structural parameters (i.e. Geometrical dimensions, Young's modulus), the imperfect measurements, the structural model, etc. During the past two decades, many authors focused their research on optimizing the configuration of sensors for the structural health monitoring. For instance, Papadimitriou (2004) used the information entropy to measure the performance of the sensors [6], Sun et Büyüköztürk (2015) proposed a discrete version of the artificial bee colony algorithm [7].

The objective of this paper consists of defining a methodology for the optimization of structural health monitoring using a predator-prey model. This optimization helps in finding the minimum number and the exact location of sensors able to detect the highest number of possible defects. The layout of the paper is as follows: Section 2 introduces the proposed methodology. Section 3 presents the Bayesian update strategy used to update the structure's information. Section 4 develops the predator-prey genetic algorithm used to find the optimal pattern of sensors. And section 5 demonstrates the applicability of the method by a numerical application on a two-story concrete frame structure.

\section{Methodology}

The methodology developed in this paper seeks to obtain an optimal configuration of sensors (i.e. quantity and location) able to identify any damage in a structure. For a particular configuration of damage, the sensitivity of the optimal configuration of sensors should be proportional to the probability of occurrence of the damage and the cost that it

*christelle.geara@net.usj.edu.lb 
engenders. Therefore, the pattern of sensors should be optimized to detect any configuration of defects. In order to do so, we propose predator-prey genetic algorithm where two populations will evolve together: (i) a population made of sensors' configurations taking the role of predators and (ii) a population made of defects' configurations taking the role of prey. For each configuration of sensors, the modal parameters are updated according to the measurements taken by sensors, before and after the occurrence of the damage, in order to identify the damaged elements. Thus, having an $a$ priori distribution of the stiffness of the structural elements, this distribution is updated by a Bayesian scheme which takes into consideration the information given by sensors and provides an a posteriori distribution of the stiffness.

In the above-proposed methodology, we assume that: (i) the structural response is obtained by measuring modal parameters (i.e. the eigenvalues and the eigenvectors) and (ii) a structural damage is defined by a loss in the stiffness of the elements.

\section{Bayesian update}

The Bayesian update allows us to consider all the uncertainties related to the degradation of the elements, the mechanical model and the accuracy of sensors measurements which are considered uncertain in our case. For a given configuration of sensors and damages, the Bayesian procedure updates the a priori distribution of the structural parameters according to the sensors' measurements [8]. If the a posteriori distribution of the structural parameters differs significantly from the $a$ priori, then a change must have occurred in the structure probably due to a damage.

The Bayesian update formula is given by:

$$
f(y \mid x)=\frac{f(x \mid y) * f(y)}{f(x)}
$$

where $y$ is the structural parameter to be updated and $x$ is the observation data, $f(y)$ is the prior distribution, $f(y \mid x)$ is the posterior distribution given the observation data $x$ and $f(x \mid y)$ is the likelihood function. In our case, we decided to use the Approximate Bayesian Computation (ABC) algorithm to calculate the a posteriori distribution since the likelihood $f(x \mid y)$ is implicit [9]. Since usually the manager of the structure has prior knowledge about the degradation of the elements and the risk parts of the structure, these information are taken into consideration in the ABC.

Thus, the $\mathrm{ABC}$ algorithm is computed as follows:

1) Postulate an a priori probability distribution of the structural parameters $(y)$;

2) Postulate an a priori probability distribution of the structural parameters change $(c)$ taking into consideration all the previous information about the degradation extent of the elements ;

3) Generate $(y)$ from the postulated a priori distribution of $(y)$;

4) Generate $(c)$ from the postulated a priori distribution of $(c)$;

5) Obtain a list of modified structural parameters $(y m)$ by multiplying each $(y)$ to its corresponding $(c)$;

6) Calculate a noisy structural response by taking into account model and sensor readings uncertainties;

7) Compare the observed and calculated structural responses using the trace of the Modal Assurance Criterion (MAC) matrix [10] defined by Eq. (2);

8) Accept $(y m)$ if the trace of the MAC is within a predefined acceptance threshold;

9) From the accepted values of $(y \mathrm{~m})$, define an a posteriori distribution of the structural parameters change;

10) If the a posteriori distribution of the structural parameters changes differs significantly from the value " 1 ", we conclude that there has been a modification in the state of the structure, probably due to a defect.

The diagonal elements of the MAC matrix to measure the correlation between two eigenvectors $\Phi_{i}$ and $\Phi^{\prime}{ }_{i}$ are given by:

$$
M A C_{i, i}=\frac{\left[\Phi_{i}^{T} \Phi_{i}^{\prime}\right]^{2}}{\left[\Phi_{i}^{T} \Phi_{i}\right]\left[\Phi \prime_{i}^{T} \Phi_{i}^{\prime}\right]}
$$

where $i$ corresponds to the vibration mode and the eigenvectors $\Phi_{i}$ and $\Phi_{i}^{\prime}$ are limited to the degrees of freedom observed by the sensors.

\section{Predator-prey genetic algorithm}

In this section, we describe the genetic optimization methodology by modeling the configurations of sensors and defects by two coevolving populations of chromosomes.

Let us consider $\boldsymbol{M}=\left\{M_{1}, M_{2}, \ldots, M_{|M|}\right\}$ the set of discretized states of the structure, $D_{i}$ the event of deciding that the structure is in state $M_{i}$ and $T_{j}$ the event stating that the real state of the structure is $M_{j}$. The Bayesian risk is then defined by the following equation:

$$
C=\sum_{k=1}^{N_{d}} \sum_{i, j=1}^{\left|M^{k}\right|} c_{i j}^{k} P\left(d_{i}^{k} \mid t_{j}^{k}\right) P\left(t_{j}^{k}\right)
$$

where $c_{i j}^{k}$ is a regret function [11-12] representing the cost incurred if we decide that the structural element $k$ is in state $M_{i}^{k}$ while in reality it is in state $M_{j}^{k}\left(C_{i i}=0\right), P\left(d_{i}^{k} \mid t_{j}^{k}\right)$ is the conditional probability to decide that the structural element 
$k$ is in state $M_{i}^{k}$ while in reality it is in state $M_{j}^{k}$ and it depends on the performance of the configuration of sensors, $P\left(t_{j}^{k}\right)$ is the a priori distribution of the real state of the structural element, and $N_{d}$ is the number of elements in the structure. We propose an approach based on the genetic optimization of two population evolving jointly: (i) a population of sensors configurations (predators) and (ii) a population of defects configurations (preys). Each configuration of sensors is made of chromosomes having a size $N_{s}$ (one gene for each degree of freedom) with genes having a value of 1 when the correspondent degree of freedom is observed by the sensors and 0 if not. Each configuration of defects is made of chromosomes having a size $N_{d}$ (equal to the number of elements in the structure) where a gene is a real value between 0 and 1 , representing the extent of the defect for a particular element. Thus, a defect gene with the value of 1 means that the element has conserved the integrality of its initial rigidity while a value of 0 means that it has lost all its rigidity.

The evaluation function of a chromosome $l$ representing the configuration of sensors must take into consideration the Bayesian risk defined in (3) and the unit price of the sensors' installation as follows:

$$
F_{l}=\frac{1}{s \times{ }^{l} N_{s}^{\prime} \sum_{k=1}^{N_{d}} \sum_{i=1}^{\left|M^{k}\right|} \sum_{j=1}^{N_{p d}} c_{i j}^{k}{ }^{l} P\left(d_{i}^{k} \mid t_{j}^{k}\right) P\left(t_{j}^{k}\right)}
$$

where ${ }^{l} N_{s}^{\prime}$ is the number of genes equal to 1 in the chromosome $l$ of sensors, $N_{p d}$ is the size of the population of prey (i.e. the states of the structure) and $S$ is the unit price of the installed sensor. The evaluation function (6) takes into consideration the probability of occurrence of a defect through $P\left(t_{j}^{k}\right)$ and favors the configurations of sensors which detect damages having a highest impact through $c_{i j}^{k}$. Therefore, the configurations of defects presenting high values of $c_{i j}^{k} P\left(t_{j}^{k}\right)$ penalize the evaluation function and encourages the chromosome of sensors to thwart their effect by minimizing ${ }^{l} P\left(d_{i}^{k} \mid t_{j}^{k}\right)$. Moreover, economical configurations of sensors are favored by $\frac{1}{S \times{ }^{l} N_{s}^{\prime}}$.

The evaluation function of a chromosome $j$ representing a configuration of the state of the structure is given by:

$$
F_{j}=\sum_{l=1}^{N_{p s}} S \times{ }^{l} N_{s}^{\prime} \sum_{k=1}^{N_{d}} \sum_{i=1}^{\left|M^{k}\right|} c_{i j}^{k}{ }^{l} P\left(d_{i}^{k} \mid t_{j}^{k}\right) P\left(t_{j}^{k}\right)
$$

where $N_{p s}$ is the size of the population of predators (configurations of sensors). This function (5) favors the states of the structure with high risks and those who are not easily detectable by sensors.

Furthermore, to avoid the imbalance between the evolution of the two populations (of predators and prey), we made it possible for a population to evolve many times in front of each evolution of the other population. This imbalance could be explained, for instance, by the ease of finding a configuration of defects non detectable by most of the chromosomes of the configuration of sensors while it is more difficult to find a configuration of sensors able to detect any configuration of defects of the actual population.

\section{Numerical application}

The proposed methodology has been applied on a simply supported concrete frame structure made of ten rectangular elements and nine nodes corresponding, in total, to 21 degrees of freedom (figure 1), with $20 \times 60 \mathrm{~cm}$ vertical elements and 20x40 cm horizontal elements. We assume that elements 1, 2, 8 and 10 are damaged, having lost $70 \%$ of their initial modulus $(33 \mathrm{GPa})$, but the damage has not been detected yet. The a priori distribution of the degradation of all the elements is supposed uniform non informative. The objective of this study is to find the best configuration of sensors (number and position) which, in our case, are supposed to be accelerometers to be placed on the degrees-of-freedom of the structure.

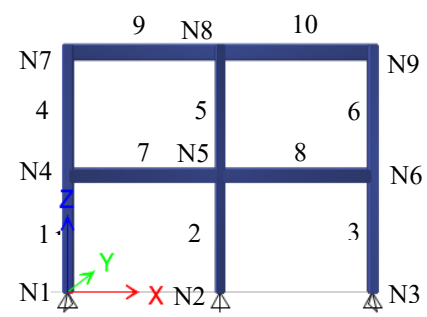

Fig. 1. Simply supported concrete frame

While applying the genetic algorithm, 100 generations of 40 individuals each were taken, with a damage chromosome of size 10 (equal to the number of elements), and a sensor chromosome of size 21 (equal to the number of the degrees-offreedom). The price of a sensor is assumed to be 2 monetary units (m.u.) and each over- or underestimation of at least $25 \%$ of an element's state is penalized by 10 m.u.

The use of the predator-prey optimization on the structure previously described led us to the optimal configuration of sensors consisting on placing two horizontal accelerometers on, respectively, the nodes (N4) and (N9), one accelerometer 
per story. This result seems to be accurate since the horizontal displacements of a structure with non-fixed nodes are more sensitive to rigidities of vertical elements, contrary to vertical displacements and rotations.

The following figures 2 and 3 show, respectively, the a posteriori distributions of the degradation rate of rigidities of columns 1 and 4 and those of beams 7 and 8, after damages have been detected by the two accelerometers. We can notice that the a posteriori distributions of the degradation rate of rigidities of damaged elements tend to value 0.3 while those of the undamaged elements tend to 1 which means that the damaged elements have only kept $30 \%$ of their initial rigidity. Thus, the results confirm the hypothesis and taken which validates the detection of damages by the accelerometers. Note that, in non-damaged elements, the distributions tend to the value 0.9 instead of 1 because of the non-informative a priori distribution taken as a uniform function from 0 to 1 with mean of 0.5 .

Moreover, supposing that damaged columns 1 and 2 and non-damaged column 3 have basically similar effects on the behavior of the structure, sensors could have been confused and detected a damage in column 3 . However, this was not the case, which confirms that the methodology can easily distinguish between cases and localize correctly any damage in a structure.

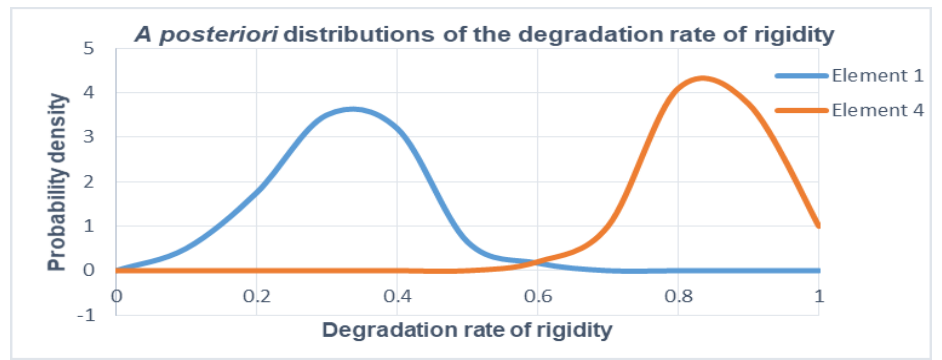

Fig. 2. A posteriori distributions of the degradation rate of rigidities of elements 1 and 4

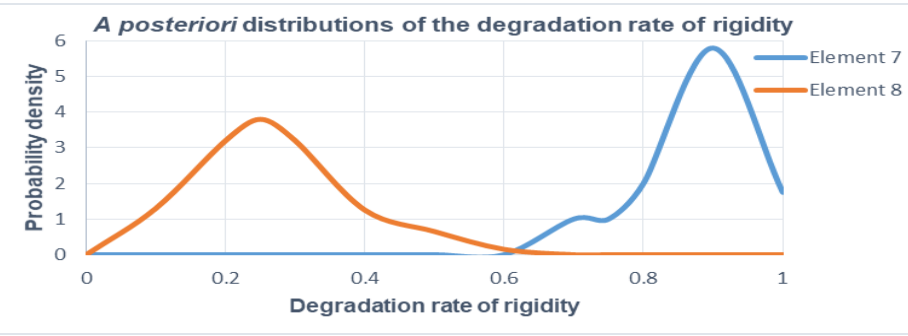

Fig. 3. A posteriori distributions of the degradation rate of rigidities of elements 7 and 8

\section{Conclusion}

In this paper, the best configuration of sensors to be placed on a damaged structure have been considered using a predatorprey optimization. An approach is proposed by applying a genetic algorithm taking into consideration a Bayesian updating of the condition states of the structural elements. This technique has been successfully applied to a concrete frame structure by providing the number and positions of sensors to be placed on it. After having analyzed the obtained results on a two-story structure, it was concluded that two horizontal accelerometers, each placed in a story, are fair enough to detect all the damages and their intensity in the structure in question.

This work was supported by the National Council for Scientific Research in Lebanon (CNRS-L).

\section{References}

1. K. Bergmeister, Monitoring and safety evaluation of existing concrete structures: State-of-art Report, 22 (2003)

2. Atkins, CSS bridge condition indicators, 3 (2002)

3. R.W. Stratt, Scool dr. stud. European Union J. 2, 67-108 (2010)

4. N. Stubbs, A literature review of recent methods for structural health monitoring systems to evaluate structural capacity and remaining service life of bridges (2011)

5. A.J. Das, A. Haldar, Int. J. Terraspace Sci. Eng., 2 (2), 101-111 (2010)

6. C. Papadimitriou, J. Sound. Vib., 28, 105-127 (2004)

7. H. Sun, O. Büyüköztürk, Smart Mater. Struct., 24, 125034 (2015) 
8. M.W. Vanik, J.L. Beck, S.K. Au, J. Eng. Mech. 126, 7 (2000)

9. R.D. Wilkinson, Stat. Appl. Genet. Mol. 12, 129-141 (2013)

10. M. Pastor, M. Binda, T. Harčarik, Procedia Eng. 48, 543-548 (2012)

11. E. Parent, J. Bernier, Le raisonnement bayésien : Modélisation et inférence (2007)

12. E.B. Flynn, M.D. Todd, Mech. Syst. Signal Pr. 24, 891-903 (2010) 